\title{
HPTLC-MS as a Neoteric Hyphenated Technique for Separation and Forensic Identification of Drugs
}

\author{
Kanak Lata Verma', Manoj Kumar², Amar Pal Singh ${ }^{1}$ \\ ${ }^{1}$ Regional Forensic Science Laboratory, Government of NCT of Delhi, New Delhi, India \\ ${ }^{2}$ Forensic Science Post Graduate Course, Department of Anthropology, University of Delhi, New Delhi, India \\ Email: kanak.dfsl@gmail.com
}

How to cite this paper: Verma, K.L., Kumar, M. and Singh, A.P. (2018) HPTLC-MS as a Neoteric Hyphenated Technique for Separation and Forensic Identification of Drugs. Journal of Analytical Sciences, Methods and Instrumentation, 8, 1-15. https://doi.org/10.4236/jasmi.2018.81001

Received: February 2, 2018

Accepted: March 26, 2018

Published: March 29, 2018

Copyright (๑) 2018 by authors and Scientific Research Publishing Inc. This work is licensed under the Creative Commons Attribution International License (CC BY 4.0).

http://creativecommons.org/licenses/by/4.0/ (c) (i) Open Access

\begin{abstract}
Drugs are traditionally been identified on basis of chromatographic-spectroscopic hyphenated techniques in instrumental analysis. Gas chromatography (GC) and Liquid chromatography (LC) hyphenated with mass spectroscopy (MS) i.e. GC-MS and LC-MS give reliable and confirmatory results in drugs identification. In the present work the novel hyphenated technique High Performance Thin Layer Chromatography-Mass Spectroscopy (HPTLC-MS) has been used. This technique provides efficient, quick and simple method for identification and separation of Narcotic drugs and psychotropic substances. The drugs under study are Papaverine, Methadone, Cocaine, Ketamine, Caffeine, Codeine, Diazepam, Thebaine, Heroin, Methamphetamine, Carbamazepine, Morphine, Narcotine and Ephedrine. The present study comprising of sixteen drugs has been carried out on CAMAG HPTLC instrument with automatic sampling. Thin layer chromatography (TLC) plates were developed in various solvent systems, scanned under TLC scanner and the results in terms of Retention Factor $\left(R_{f}\right.$ value) and UV spectrum $(\lambda \max )$ are presented in the manuscript. Using hyphenated technique of HPTLC-MS (MS 2020 SHIMADZU) spots of these drugs from TLC plate was lifted with CAMAG TLC-MS interface and confirmed by the mass spectrum of the individual drugs by their $\mathrm{m} / \mathrm{z}$ values thus delivering fast and accurate confirmatory result on the TLC plate.
\end{abstract}

\section{Keywords}

Narcotic Drugs, Psychotropic Substances, HPTLC-MS, Chromatography, Mass Spectroscopy, Hyphenated Techniques, Forensic Science 


\section{Introduction}

India geographical location makes it proximal to drug problem as India is sandwiched between the world's two largest areas of illicit opium production, the golden crescent i.e. Pakistan, Afghanistan, Iran and the golden triangle i.e. Vietnam, Laos, Thailand, Myanmar. The proximity has traditionally been viewed as a source of vulnerability, since it has made India both a destination and a transit route for opiates, cannabis, amphetamines and synthetic drugs produced in these regions [1]. This fact defines drug trafficking trends in the subcontinent. International drug trafficking is not just a trade for financial purposes but provides funding for cross border terrorisms which is called drug terrorism. The United Nations Conference for the Adoption of a Single Convention on Narcotic Drugs met at United Nations Headquarters from 24 January to 25 March 1961 to reduce the number of international treaty organs exclusively concerned with control of narcotic drugs, and to make provision for the control of the production of raw materials of narcotic drugs. The United Nations Conference for the Adoption of a Protocol on Psychotropic Substances met in Vienna from 11 January to 21 February 1971 and determined to prevent and combat abuse of such substances and the illicit traffic to which it gives rise. United Nations Convention against Illicit Traffic in Narcotic Drugs and Psychotropic Substances, 1988 provides comprehensive measures against drug trafficking, including provisions against money laundering and the diversion of precursor chemicals. It provides for international cooperation through, for example, extradition of drug traffickers, controlled deliveries and transfer of proceedings [2]. On part of India, government agencies seize tons of Heroin along with other narcotic drugs and psychotropic substances under NDPS Act, 1985. During 2015, $1687 \mathrm{kgs}$ of Opium, $1416 \mathrm{~kg}$ of Heroin, 94,403 kg of Ganja, $3349 \mathrm{~kg}$ of Hashish, $113 \mathrm{~kg}$ of Cocaine, $827 \mathrm{~kg}$ of Ephedrine/Pseudo-Ephedrine, $211 \mathrm{~kg}$ of Ketamine etc. were seized by various agencies. Illicit Opium poppy cultivation spread over 3461 acres \& illicit cannabis cultivation spread over 818 acres were destroyed all over the country [3]. The NDPS Act provides a strong system to prevent illegal drug trafficking and prevention over clandestine laboratories in India. The NDPS (The Narcotic Drugs and Psychotropic substances Act, 1985) act is provisions for the control and regulation of operations relating to An Act to consolidate and amend the law relating to narcotic drugs, to make stringent narcotic drugs and psychotropic substances [1], to provide for the forfeiture of property derived from, or used in, illicit traffic in narcotic drugs and psychotropic substances, to implement the provisions of the International Convention on Narcotic Drugs and Psychotropic Substances and for matters connected therewith [4]. In spite of these stringent national and international efforts and law, drug seizure data show that illegal drug manufacturing in clandestine laboratories and trafficking is flourishing. The penalty for manufacturing, illegal consumption, holding and trafficking depends upon nature of substance seized from the culprit. So, complete characterization and identification of seized Narcotics and Psychotropic drugs is of ut- 
most importance to penalise the culprit with proper punishment. Drugs identification and separation is traditionally being done by chromatography [5] which was discovered by M. Tswett in 1906 and in 1938 Izmailov and Shreiber introduced TLC with some modifications. Chromatography is "the separation of two or more compounds by distribution between two phases. One is stationary phase and other is mobile phase" [6]. These two phases can be solid-liquid, liquid-liquid and gas-liquid. The principle behind the work of this method is that different types of compounds with different solubility, adsorption and polarity to the two phases by which they are partitioned. The compounds to be retained on the stationary phase are more interacted with it while the compounds to be moving carried along by the mobile phase. The rates of migration for each component on the system is depends on the degree of the compounds of mixture are adsorbed by the stationary phase and their degree of solubility on the mobile phase. The stronger the adsorption by stationary phase, the slower the compound will move with the mobile phase. Due to which the migration distance for each substance is found to be different and the components of the mixtures get separated. The migration distance of substances on TLC chromatograms are generally fixed as Rf value. The types of chromatography is divided into few types which are gas chromatography (GC), high performance liquid chromatography (HPLC), thin layer chromatography (TLC) and column chromatography (CC). Different types of chromatography make the task of identification firm, easy and reliable. Since in forensic science laboratories drugs are identified and separated by TLC, HPTLC, GC-FID and known hyphenated techniques which are GC-MS, LC-MS. Drugs are traditionally been identified on basis of chromatographic-spectroscopic hyphenated techniques in instrumental analysis. Gas chromatography and Liquid chromatography hyphenated with mass spectroscopy i.e. GC-MS and LC-MS give very reliable results in drugs identification. In the present work the novel hyphenated technique High Performance Thin Layer Chromatography-Mass Spectroscopy (HPTLC-MS) has been used to develop a new method for identification and separation of drugs with High Performance Thin Layer chromatography hyphenated with Mass spectroscopy. This technique provides efficient, quick and simple method for identification and separation of Narcotic drugs and psychotropic substances. High Performance Thin Layer Chromatography (HPTLC) is the most advanced form of TLC and comprises the use of chromatographic layers of utmost separation efficiency and the employment of state of the art instrumentation for all steps in the procedure: precise sample application, standardized reproducible chromatogram development and software controlled evaluation. HPTLC is an entire concept that includes a widely standardized methodology based on a scientific facts as well as the use of validated methods for quantitative and qualitative analysis. HPTLC meets all quality requirements of today's analytical labs. The possibility of visual evaluation of the separated samples on the plate is one of the most valuable aspects of TLC [7]. Advantages of HPTLC include that the technique is simple to 
learn, operate, several analysis works could be done on same time, is a fast and economic technique. Visual detection possible as it is an open system and at the same time there is availability of great range of stationary phases with unique selectivity of mixture components. The plates do not require regeneration as they are disposable. Availability to choose mobile phase is not restricted by low UV transparency or the need of ultra-high purity. Corrosive and UV absorbing mobile phase can be employed. There is no possibility of interference from previous analysis as fresh stationary and mobile phase is used for each analysis, so no carry over and no contamination. TLC scanner provides the output to a system in terms of Retention factors of the spot, height and area of the peak for quantification and absorbance in UV-Visible spectrum [8] for substance identification. UV and visible spectrophotometry find their primary application in quantitative analysis. The scope of absorption spectroscopy can be extended significantly by the use of colour reactions, often with a concomitant increase in sensitivity and/or selectivity. Such reactions are used to modify the spectrum of an absorbing molecule so that it can be detected in the visible region, well separated from other interfering components in the UV spectrum. Moreover, chemical modification can be used to transform an otherwise non-absorbing molecule into a stable derivative that possesses significant absorption [9]. The whole instrument becomes very efficient for substance identification having output as mass spectrum of the substance when Mass Spectroscopy is hyphenated with the HPTLC instrument and the qualitative analysis is confirmatory. A mass spectrometer works by generating charged molecules or molecular fragments either in a high vacuum or immediately before the sample enters the high-vacuum region. Instruments typically maintain vacuums of about $10-6 \mathrm{mmHg}$, since ionised molecules have to be generated in the gas phase to be able to manipulate those using magnetic or electrostatic fields. In classic mass spectrometry (MS) only one method could produce the charged gaseous molecules, but now quite a number of alternatives are available. Once the molecules are charged and in the gas phase, they can be manipulated by the application of either electric or magnetic fields to enable the determination of their relative molecular mass and the relative molecular mass of any fragments produced by the molecules breaking up [10] [11].

In the present work, drugs examined under HPTLC-MS (CAMAG instrument) are Methadone, Papaverine, Cocaine, Ketamine, Phenobarbital, Caffeine, Codeine, Acetaminophen, Diazepam, Thebaine, Heroin, Methamphetamine, Carbamazepine, Narcotine, Morphine and Ephedrine. Most of these drugs are often encountered in seized samples of drugs. These drugs are used illicitly in various forms and through multiple routes. Serious risks include opioid abuse or a decreased effort to breathe [12] [13] [14]. Ketamine, a phencyclidine derivative, is a unique dissociative anaesthetic that produces amnesia and intense analgesia [15]. Phenobarbital is an inexpensive anticonvulsant, and will therefore remain important in developing countries. In the developed world, where cost is less of a determining factor, it is less attractive than its better-tolerated succes- 
sors [16]. Caffeine is one of the world's most widely ingested chemicals, The first use of coffee beans occurred in Ethiopia as far back as the ninth century [17]. Codeine is an alkaloid found in very low concentrations in opium; it is now derived from morphine. Codeine is frequently administered in combination with acetaminophen, butalbital, and caffeine [18] Acetaminophen (known as paracetamol) is the most commonly administered over-the-counter analgesic, [19]. The second benzodiazepine to be marketed, diazepam, was approved for human use in the early 1960s and has sedative, hypnotic, muscle relaxant and anticonvulsant properties [20]. Thebaine (paramorphine), also known as codeine methyl enol ether, is an opiate alkaloid and at high doses, it causes convulsions similar to strychnine poisoning [21]. Heroin is an opioid drug made from morphine, a natural substance taken from the seed pod of the Asian opium poppy plant. Some people mix heroin with crack cocaine, called a speedball [22]. Methamphetamine is a psycho-stimulant that is similar in chemical structure to amphetamine but has more profound effects on the central nervous system. Like cocaine, methamphetamine ingestion produces stimulation and similar feelings of euphoria; however, methamphetamine has a longer duration of action $(6-8 \mathrm{~h}$ after a single dose) [23]. Carbamazepine is an anticonvulsant with structural similarity to the cyclic antidepressants [24]. Narcotine is a naturally occurring, opium-isoquinoline alkaloid that is related to papaverine. It acts centrally as a cough suppressant and has actions and uses similar to dextromethorphan. Morphine is a pain medication of the opiate type which is found naturally in a number of plants and animals. It acts directly on the central nervous system (CNS) to decrease the feeling of pain. Potentially serious side effects include a decreased respiratory effort and low blood pressure. Morphine has a high potential for addiction and abuse. If the dose is reduced after long-term use, withdrawal may occur. Common side effects include drowsiness, vomiting, and constipation. Caution is advised when used during pregnancy or breast feeding, as morphine will affect the baby [25]. Ephedrine is one of the most commonly used non catecholamine sympathomimetic drugs in the preoperative period [26].

Thin layer chromatography (TLC) and gas chromatography-mass spectroscopy (GC-MS) have been reportedly used to confirm the presence of heroin and its metabolite 6-monoacetyl morphine. The presence of 6-monoacetyl morphine determines definite heroin exposuree [27]. The application of high performance thin layer chromatography-bioautography in tandem with mass spectrometry was investigated for the rapid identification of antimicrobial and anti-quorum sensing (anti-QS) compounds [28]. Simultaneous Quantification of Methadone, Cocaine, Opiates, and Metabolites in Human Placenta by Liquid Chromatography-Mass Spectroscopy has been done [29]. HPTLC method for simultanoeus determination of alprazolam and methyl paraben in tablet dosage form has been carried out by Sagar B. Wankhede et al. [30]. Hui Qiao et al. have reported a Quantitative Determination of Methadone and its Metabolite (EDDP) in Dry Blood Spot by LC-MSMS [31]. Ketamine popularly known as date-rape drug 
abused in rave parties and cause of the crimes against the women and determination of ketamine and its residues in non-biological exhibits in cases related to drug facilitated sexual assaults by LC-MS/MS has been reported [32].

\section{Experimental Set-Up}

To execute a new hyphenated technique for separation and forensic identification of drugs with the following steps:

1) To find out solvent system using HPTLC for the following drugs

Methadone, Papaverine, Cocaine, Ketamine, Phenobarbital, Caffeine, Codeine Acetaminophen, Diazepam, Thebaine, Heroin, Methamphetamine, Carbamazepine, Narcotine, Morphine and Ephedrine.

2) To separate the drugs and find out the retention factor using HPTLC for each drug in its respective solvent systems in particular conditions.

3) Qualitative analysis to find out maximum absorbance and UV spectrum for each drug in its particular condition.

4) To confirm the identification of drugs by hyphenated mass technique by obtaining its mass spectrum and analysis of the peaks obtained.

\section{Materials and Methods}

Chemicals And Reagents: Standard drugs include Methadone, Papaverine, Cocaine, Ketamine, Phenobarbital, Caffeine, Acetaminophen, Diazepam, The baine, Methamphetamine, Carbamazepine, and Ephedrine Heroin, Codeine, Narcotine, Morphine (From Indian Pharmacopoeia Commission, Sigma-Aldrich and Government Opium and Alkaloid Works, Neemuch, India).

Reagents of sample preparation-Millipore water, Ethanol, Methanol (HPLC Grade), Chloroform (HPLC Grade), Reagents for Mobile phase preparation-Chloroform (HPLC Grade), Methanol (HPLC Grade), Ammonia, Ethyl acetate (HPLC Grade),Carrier Solvent for MS interface-Methanol (HPLC Grade), Acetonitrile (HPLC Grade), Pre coated $10 \times 10$ Silica gel 60 F254 TLC plates, Micro-pipette, Spatula, Test tubes, TLC Development Chamber (Camag).

\section{Extraction Method}

\section{1) Preparation of standard samples}

Standard solutions of drugs were prepared using HPLC Grade solvents. Solvent selection for standard solutions was done according to the solubility [33] of drugs. Standard solutions of drugs Methadone, Cocaine, Phenobarbital, Codeine, Acetaminophen, Methamphetamine, Carbamazepine, Morphine and Ephedrine were taken in $5 \mathrm{ml}$ volumetric flask and ethanol was added to each of the volumetric flask and volume was extended up to $5 \mathrm{ml}$ mark. Standard solutions for Papaverine, Caffeine, Diazepam, Thebaine, Heroin and Narcotine were prepared in $5 \mathrm{ml}$ Chloroform of HPLC Grade. Ketamine standard was prepared in 5 $\mathrm{ml}$ Methanol of HPLC Grade.

2) Preparation of developing solvents (mobile phase) 
Three different Mobile phases were prepared for TLC development as shown in Table 1. For selection of mobile phase drugs were categorized into two categories. One is basic and other is acidic.

\section{a) Basic drugs}

Methadone, Papaverine, Cocaine, Ketamine, Caffeine, Codeine, Diazepam, Thebaine, Heroin, Methamphetamine, Carbamazepine, Narcotine, Morphine \& Ephedrine.

\section{b) Acidic drugs}

Phenobarbital \& Acetaminophen.

\section{Instrumental Methods}

Analysis of the drugs was performed using CAMAG HPTLC instrument coupled with MS (MS 2020 SHIMADZU) by CAMAG TLC-MS interface and system was controlled with winCATS CAMAG HPTLC Software. The fully automatic sample application device used was ATS 4 (CAMAG Automated TLC Sampler 4) fitted with a $25 \mu \mathrm{l}$ Hamilton micro syringe and connected to a nitrogen supply. Four narrow band spots of $6.0 \mathrm{~mm}$ length were applied above $8 \mathrm{~mm}$ on pre coated $10 \times 10$ Silica gel 60 F254 TLC plate for each drug sample of different concentration $(2 \mu \mathrm{l}, 5 \mu \mathrm{l}, 10 \mu \mathrm{l}, 2 \mu \mathrm{l})$. Methanol was used as solvent and Nitrogen gas provided pressure for spraying the sample for application ensuring homogenous distribution of the sample across the entire length of application zone. After application plate was dried for 10 minutes at room temperature $\left(25^{\circ}\right.$ Celsius).

1) Sample application

Sampling Type-Automatic Spraying, Instrument Used-CAMAG HPTLC

No. of spots $-4(2 \mu \mathrm{l}, 5 \mu \mathrm{l}, 10 \mu \mathrm{l}, 2 \mu \mathrm{l})$, Band Length $-6 \mathrm{~mm}$, Plate Size $-10 \times$ 10.

Solvent used-Methanol HPLC Grade, Pressure gas-Nitrogen, Drying-At $25^{\circ} \mathrm{C}$ for 10 minutes, Starting spot position $-8 \mathrm{~mm}$, Solvent front position -80 $\mathrm{mm}$.

\section{2) Development}

After precise sample application, the chromatograms were developed in their respective solvent system as specified in Table 2. Development of HPTLC plate was done in $10 \times 10$ twin trough chamber saturated with mobile phase for 15 minutes. Solvent front was moved to a migration distance of $80 \mathrm{~mm}$ of the plate and plate was dried at room temperature. Three types of mobile phase were used for better separation.

Table 1. Solvent system used for TLC development.

\begin{tabular}{cc}
\hline SOLVENT SYSTEM & SOLVENT USED (10 ml) \\
\hline A & CHLOROFORM:METHANOL (9:1) \\
B & METHANOL:AMMONIA (10:0.15) \\
C & ETHYL ACETATE:METHANOL:AMMONIA (8.5:1:0.5) \\
\hline
\end{tabular}


Table 2. Drugs and their respective solvent system.

\begin{tabular}{ccc}
\hline S. No. & NAME OF THE DRUG & SOLVENT SYSTEM \\
\hline 1 & METHADONE & A \& B \\
2 & PAPAVERINE & A \\
3 & COCAINE & A \\
4 & KETAMINE & A \\
5 & PHENOBARBITAL & C \\
6 & CAFFEINE & A \\
7 & CODEINE & A \& B \\
8 & ACETAMINOPHEN & C \\
9 & DIAZEPAM & A \\
10 & THEBAINE & A \\
11 & HEROIN & A \\
12 & METHAMPHETAMINE & B \\
13 & CARBAMAZEPINE & A \\
14 & NARCOTINE & A \\
15 & MORPHINE & B \\
16 & EPHEDRINE & B \\
\hline
\end{tabular}

*Solvent systems: Refer to Table 1 .

\section{3) Documentation}

Reproducibly acquire and preserve best quality images of TLC/HPTLC chromatograms under different illuminations this high-end imaging and documentation system is now available. With its new digital CCD camera a maximum resolution of $82 \mu \mathrm{m}$ on the plate is obtained. For electronic image acquisition the camera-like the human eye-captures polychromatic visible light. Under white light illumination it is the light reflected from the layer background. Under long-wavelength UV light $(366 \mathrm{~nm})$ it is the light emitted by fluorescent substances. When short-wavelength UV light $(254 \mathrm{~nm})$ is used, substances absorbing UV 254 appear as dark zones, provided the layer contains a fluorescence indicator. Chromatograms on the plate were visualised and photographed for documentation under UV $254 \mathrm{~nm}$, UV $366 \mathrm{~nm}$ and in white light with 85 point exposure. The chromatograms were saved and documented.

\section{4) TLC scanner}

WIN CATS controls the CAMAG TLC Scanner 4 and enables quantitative evaluation of the generated densitometry data. To determine the substance concentration in a sample, five different quantification functions (e.g. linear and polynomial) are available. Several scanning steps (e.g. scanning the plate after development) and up to five different evaluations can be performed (single wavelength, multiple wavelengths or a combination of measurements in absorption and fluorescence detection mode). TLC plate was scanned under TLC Scanner. 
Spots were localised by automatic scanning and Rf (Retention factor) of each spot was calculated and substance assignment was done in the evaluation system. After proper substance assignment TLC plate was further scanned under UV (200 - $400 \mathrm{~nm}$ ) using Deuterium lamp. The output from the spectrum for each drug in UV light was obtained, analysed and recorded in Table 3.

\section{5) Lifting spots with camag TLC-MS interface}

Spot were marked after visualising under UV light and lifted with CAMAG TLC-MS interface. Carrier solvent i.e. Methanol and Acetonitrile (HPLC Grade) for drug was taken into source solvent container. The SCHIMADZU 2020 Mass Spectrometer was controlled with Lab Solutions software. The chromatogram zones are eluted from the HPTLC plate with suitable solvents as shown in Table 4 with the flow speed appropriate for the LC-MS system. The round elution head is used for circular zones and the oval elution head for zones in the form of bands. After extraction of the elute is either transferred online to the mass spectrometer or collected in a sample vial for further offline analysis. The CAMAG TLC-MS Interface 2 features a modified elution head and an easily accessible, exchangeable filter, arranged in front of the valve. Cleaning is facilitated as compared to the previous version, making it highly efficient. By pushing a button,

Table 3. Retention factor and maximum absorbence of drug.

\begin{tabular}{|c|c|c|c|c|}
\hline S. No. & NAME OF THE DRUG & $\begin{array}{l}\text { SOLVENT } \\
\text { SYSTEM }^{*}\end{array}$ & $\begin{array}{l}\text { RETENTION } \\
\text { FACTOR }\end{array}$ & $\begin{array}{l}\lambda \max \\
(\mathrm{nm})\end{array}$ \\
\hline \multirow{2}{*}{1} & \multirow{2}{*}{ METHADONE } & A & 0.15 & 200 \\
\hline & & $\mathrm{B}$ & 0.48 & 200 \\
\hline 2 & PAPAVERINE & A & 0.91 & 243 \\
\hline 3 & COCAINE & A & 0.44 & 234 \\
\hline 4 & KETAMINE & A & 0.85 & 200 \\
\hline 5 & PHENOBARBITAL & $\mathrm{C}$ & 0.63 & 200 \\
\hline 6 & CAFFEINE & A & 0.74 & 275 \\
\hline \multirow[b]{2}{*}{7} & \multirow[b]{2}{*}{ CODEINE } & A & 0.25 & 212 \\
\hline & & B & 0.43 & 209 \\
\hline 8 & ACETAMINOPHEN & $\mathrm{C}$ & 0.68 & 246 \\
\hline 9 & DIAZEPAM & A & 0.93 & 231 \\
\hline 10 & THEBAINE & A & 0.39 & 285 \\
\hline 11 & HEROIN & A & 0.47 & 206 \\
\hline 12 & METHAMPHETAMINE & B & 0.31 & 205 \\
\hline 13 & CARBAMAZEPINE & A & 0.78 & 286 \\
\hline 14 & NARCOTINE & A & 0.87 & 217 \\
\hline 15 & MORPHINE & B & 0.41 & 210 \\
\hline 16 & EPHEDRINE & $\mathrm{B}$ & 0.36 & 205 \\
\hline
\end{tabular}

*Solvent systems: Refer to Table 1. 
Table 4. Drugs with their lifting solvent.

\begin{tabular}{|c|c|c|c|}
\hline IUPAC NAME & $\begin{array}{l}\text { MOLECULAR } \\
\text { WEIGHT }\end{array}$ & EVENT & $\begin{array}{l}\text { LIFTING } \\
\text { SOLVENT }\end{array}$ \\
\hline 6-Dimethylamino-4,4-diphenyl-3-heptanone & 309.4 & 1 & M \\
\hline 1-[(3,4-Dimethoxyphenyl)methyl]-6,7-dimethoxyisouinoline & 339.4 & 1 & M \\
\hline Methyl-[1R-exo,exo)]-3-(benzoyloxy)-8-methyl-8-azabicyclo[2.2.1]-octane-2-carboxylate & 303.4 & 1 & M \\
\hline 2-(2-chlorophenyl)-2-(methylamino)cyclohexanone & 237.7 & 1 & M \\
\hline 5-Ethyl-5-phenyl-2,4,6(1H,3H,5H)-pyirimidinetrione & 232.2 & 2 & M \\
\hline 3,7-Didehydro-1,3,7-trimethyl- $1 H$-purine-2,6-dione & 194.2 & 2 & M \\
\hline 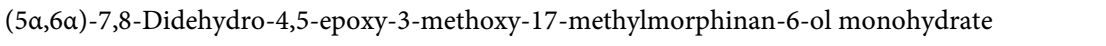 & 294.4 & 1 & M \\
\hline $\mathrm{N}$-(4-Hydroxyphenyl)acetamide & 151.2 & 2 & M \\
\hline 7-Chloro-1,3-dihydro-1-methyl-5-phenyl-2 $H$-1,4-benzodiazepin-2-one & 284.7 & 1 & A \\
\hline (5a)-6,7,8,14-Tetradehydro-4,5-epoxy-3,6-dimethoxy-17-methylmorphina & 311.4 & 1 & M \\
\hline (5a,6a)-7,8-Didehydro-4,5-epoxy-17-methylmorphinan-3,6-dioldiacetate & 369.4 & 1 & A \\
\hline$(\alpha S)-N, \alpha$-Dimethylbenzeneethanamine & 149.2 & 2 & M \\
\hline 5 H-Dibenz $[b, f]$ azepine-5-carboxamide & 236.3 & 2 & A \\
\hline $\begin{array}{l}\text { (3S)-6,7-Dimethoxy-3-[(5R)-5,6,7,8-tetrahydro-4-methoxy-6-methyl-1,3-dioxolo[4,5-g]isoquin } \\
\text { oline-5-yl]-1(3H)-isoenzofuranone }\end{array}$ & 413.4 & 1 & A \\
\hline 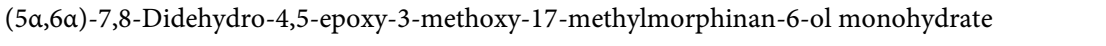 & 285.3 & 2 & A \\
\hline$(\alpha \mathrm{R})-\alpha[(1 \mathrm{~S})-1-($ Methylamino)ethyl]benzenemethanol hemihydrate & 165.2 & 1 & M \\
\hline
\end{tabular}

$\mathrm{M}=$ Methanol; A = Acetonitrile; Even 1: Positive Ionisation; Even: Negative Ionisation.

the elution path is cleaned of matrix particles with compressed air, increasing the lifetime of the filter and preventing the system from becoming blocked. These filters can be easily replaced without any modification to the elution head.

The flow rate for mobile solvent for lifting up the spot from TLC was kept at $0.5 \mathrm{ml}$ per minute and flow of Nebulising gas was kept at $1.5 \mathrm{~L} / \mathrm{min}$. The ionization mode was electrospray ionization with chemical ionization. DL Temperature was $250^{\circ} \mathrm{C}$ and detector voltage was kept at $0.95 \mathrm{KV}$. Spectra were acquired in according to the mass of drugs in mass/charge $(\mathrm{m} / \mathrm{z})$ ratio range. Detection of ions was done at both events positive and negative ion mode. The base peak and mass peak was observed and recorded in Table 5 .

\section{Results}

Developed chromatograms were scanned under CAMAG TLC Scanner and output was Chromatogramed and UV spectrum display was recorded in observations. From the chromatogram and UV spectra for all tracks of drugs the output received was recorded in Table 3 in terms of Retention Factor (Rf value) and maximum absorbance pertaining to the conditions followed in materials and methods. The data received was sufficient for separation and identification of each drug. On further processing, when the spot was lifted with Camag 
Table 5. MS peak output of drugs.

\begin{tabular}{|c|c|c|c|c|c|}
\hline S. No. & $\begin{array}{l}\text { NAME OF THE } \\
\text { DRUG }\end{array}$ & IUPAC NAME & $\begin{array}{l}\text { MOLECULA } \\
\text { R WEIGHT }\end{array}$ & EVENT & $\begin{array}{l}\text { Peak detected in } \\
\text { MS ( } m / z \text { ratio) }\end{array}$ \\
\hline 1 & METHADONE & 6-Dimethylamino-4,4-diphenyl-3-heptanone & 309.4 & 1 & 310 \\
\hline 2 & PAPAVERINE & $\begin{array}{l}\text { 1-[(3,4-Dimethoxyphenyl)methyl]-6,7-dimethoxyiso } \\
\text { uinoline }\end{array}$ & 339.4 & 1 & 340 \\
\hline 3 & COCAINE & $\begin{array}{l}\text { Methyl-[1R-exo,exo)]-3-(benzoyloxy)-8-methyl-8- } \\
\text { azabicyclo[2.2.1]-octane-2-carboxylate }\end{array}$ & 303.4 & 1 & $326^{*}, 304^{* *}$ \\
\hline 4 & KETAMINE & 2-(2-chlorophenyl)-2-(methylamino)cyclohexanone & 237.7 & 1 & 238 \\
\hline 5 & PHENOBARBITAL & 5-Ethyl-5-phenyl-2,4,6(1H,3H,5H)-pyirimidinetrione & 232.2 & 2 & 231 \\
\hline 6 & CAFFEINE & 3,7-Didehydro-1,3,7-trimethyl-1 $H$-purine-2,6-dione & 194.2 & 2 & $219^{*}, 193^{* *}$ \\
\hline 7 & CODEINE & $\begin{array}{l}\text { (5a,6a)-7,8-Didehydro-4,5-epoxy-3-methoxy-17-met } \\
\text { hylmorphinan-6-ol monohydrate }\end{array}$ & 294.4 & 1 & $322^{*}, 293^{* *}$ \\
\hline 8 & ACETAMINOPHEN & $\mathrm{N}$-(4-Hydroxyphenyl)acetamide & 151.2 & 2 & $182^{\star}, 150^{* *}$ \\
\hline 9 & DIAZEPAM & $\begin{array}{l}\text { 7-Chloro-1,3-dihydro-1-methyl-5-phenyl-2 } H \text {-1,4-be } \\
\text { nzodiazepin-2-one }\end{array}$ & 284.7 & 1 & $339^{*}, 285^{* *}$ \\
\hline 10 & THEBAINE & $\begin{array}{l}\text { (5a)-6,7,8,14-Tetradehydro-4,5-epoxy-3,6dimethoxy- } \\
\text { 17-methylmorphina }\end{array}$ & 311.4 & 1 & 312 \\
\hline 11 & HEROIN & $\begin{array}{l}\text { (5a,6a)-7,8-Didehydro-4,5-epoxy-17-methylmorphin } \\
\text { an-3,6-dioldiacetate }\end{array}$ & 369.4 & 1 & $411^{*}, 369^{* *}$ \\
\hline 12 & $\begin{array}{l}\text { METHAMPHETAMI } \\
\text { NE }\end{array}$ & $(\alpha \mathrm{S})$-N, $\alpha$-Dimethylbenzeneethanamine & 149.2 & 2 & 149 \\
\hline 13 & CARBAMAZEPINE & 5H-Dibenz $[b, f]$ azepine-5-carboxamide & 236.3 & 2 & $291^{\star} 237^{\star *}$ \\
\hline 14 & NARCOTINE & $\begin{array}{l}\text { (3S)-6,7-Dimethoxy-3-[(5R)-5,6,7,8-tetrahydro-4-me } \\
\text { thoxy-6-methyl-1,3-dioxolo[4,5-g]isoquinoline-5-yl]- } \\
\text { 1(3H)-isoenzofuranone }\end{array}$ & 413.4 & 1 & $477^{\star}, 414^{* *}$ \\
\hline 15 & MORPHINE & $\begin{array}{l}\text { (5a,6a)-7,8-Didehydro-4,5-epoxy-3-methoxy-17-met } \\
\text { hylmorphinan-6-ol monohydrate }\end{array}$ & 285.3 & 2 & $316^{*}, 284^{* *}$ \\
\hline 16 & EPHEDRINE & $\begin{array}{l}\text { ( } \alpha \mathrm{R})-\alpha[(1 \mathrm{~S})-1-(\text { Methylamino)ethyl] benzenemethanol } \\
\text { hemihydrate }\end{array}$ & 165.2 & 1 & 166 \\
\hline
\end{tabular}

*Base peak; ${ }^{*}$ Actual molecular mass peak.

TLC-MS interface, the Chromatogram and Mass spectrum was obtained as output which was recorded in observations. From the chromatogram and Mass Spectrum of all the drugs the output received was their base peak or mass peak indicating the exact molecular mass of the substance which is recorded in Table 4. The data received was sufficient for confirmation of the identity of the drug as it shows the exact molecular weight.

Each of the drugs was separated with good resolution using different mobile phases. The aim of the study of separating the drugs was done using three suitable solvent systems for all the sixteen drugs which would help in rapid screening of these drugs exhibit received for chemical examination in the forensic science laboratory for queries pertaining to identification and detection of all these drugs. These sixteen drugs are the most frequently encountered during investi- 
gation by toxicologists. The solvent system $\mathrm{A}$ is found to be most versatile which could separate eleven of the sixteen drugs which are Methadone, Papaverine, Cocaine, Ketamine, Caffeine, Codeine, Diazepam, Thebaine, Heroin, Methamphetamine, Carbamazepine, Narcotine, Morphine and Ephedrine. Solvent system B was found to be best for good resolution for Methamphetamine, Morphine and Ephedrine. Codeine and Methadone also gave good results in context of the Rf values in solvent system B $(0.43,0.48)$ as compared to solvent system A $(0.25,0.15)$ respectively. Solvent system $C$ was best for separation of acidic drugs i.e. Phenobarbital and Acetaminophen with Rf 0.63 and 0.68 respectively The UV spectrum shown by the drugs and the maximum absorbance in the particular conditions makes it possible to identify each and every drug. The Rf and $\lambda \max$ for all the drugs in their respective solvents is given in Table 3 . In the present study HPTLC system instrument has been used. Automated spotting, detection and scanning of the TLC plate makes this technique excellent for rapid, efficient and reliable screening of drugs especially for the forensic science laboratory where large number of exhibits are to be examined with in a stipulated period of time to counter the pendency of cases. The last and final aim of the study was achieved as the mass data is observed which is tabulated in Table 5. The analysis is done in two modes, positive ion mode i.e. event 1 and negative ion mode i.e. event 2. In negative ion mode i.e. EVENT 2 with electrospray ionisation (ESI) and atmospheric pressure chemical ionization (APCI) of drugs showed major ions [M-H]- at $m / z 231$ for Phenobarbital, $m / z 219$ and $m / z 193$ for Caffeine, $m / z 182$ and $m / z 150$ for Acetaminophen, $m / z 149$ for Methamphetamine and $m / z 316$ and 284 for Morphine. Positive ion mode i.e. EVENT 1 with electrospray ionisation (ESI) and atmospheric pressure chemical ionization (APCI) of drugs showed major ions $[\mathrm{M}-\mathrm{H}]+$ at $\mathrm{m} / \mathrm{z} 310$ for Methadone, $\mathrm{m} / \mathrm{z} 340$ for Papaverine, $m / z 326$ and 304 for Cocaine, $m / z 238$ for Ketamine, $m / z 322$ and 293 for Codeine, $\mathrm{m} / z 339$ and 285 for Diazepam, $\mathrm{m} / \mathrm{z} 312$ for Thebaine, $\mathrm{m} / \mathrm{z}$ 411 and 369 for Heroin, $m / z 477 \& 413$ for Narcotine and 166 for Ephedrine. Base peak of mass spectrum of drugs Methadone, Papaverine, Ketamine, Phenobarbital, Thebaine, Methamphetamine and Ephedrine showed their actual molecular mass with +1 or -1 addition to their base peak in terms of $m / z$. So, all the drugs standards were analysed for both the events and appropriate event was selected by the analysing peaks detected and actual molecular mass of the substance. Rest of the drugs which are Cocaine, Caffeine, Codeine, Acetaminophen, Diazepam, Heroin, Carbamazepine, Narcotine and Morphine showed multiple peaks along with the actual molecular mass of the drug.

\section{Conclusions}

The aims and objectives of the study have been addressed successfully. The present study has been conducted in view of the drugs which are often encountered by the toxicologists and forensic chemical examiners during their routine work. By using the HPTLC-MS technique the sixteen drugs which are Papave- 
rine, Methadone, Cocaine, Ketamine, Caffein, Codeine, Diazepam, Thebaine, Heroin, Methamphetamine, Carbamazepine, Morphine, Narcotine and Ephedrine are successfully separated with high performance thin layer chromatography and identified by their UV absorbance and mass spectrometry. The hyphenated technique of HPTLC-MS is an exemplary technique where in scientist has the opportunity to play with a number of solvent systems and optimise/validate his method best suited to the compound in question with in a very short time. It is a rapid method where spot can be directly lifted from developed TLC plate and extracted using TLC-MS interface. The whole exercise is completed within 10 to 20 minutes. The compound in question is identified by confirmation of its molecular weight $\mathrm{m} / \mathrm{z}$ value. The base peak present other than the actual molecular weight and the variation of \pm 1 is due to adduct formation of the drug sample with its solvent. The technique has opened door for scientists to move towards economic and clean technology wherein minimum amount of solvents are used. When the spot is directly lifted from the plate for its mass analysis, it reduces the chances of contamination and cross contamination which is major issue with columns used in GC-MS technology.

The future course of action pertaining to this study will be to explore the possibility of developing the single common solvent system for all these drugs so that the challenge before the forensic scientist pertaining to time constraint and resources is addressed successfully. The drugs and substances other than these sixteen could also be taken for the same study. The technique could be used for the quantitative analysis in further course of the studies.

\section{Acknowledgements}

The authors gratefully acknowledge Director, Forensic Science Laboratory and Head of Office Regional Forensic Science Laboratory, Chanakya Puri, Government of National Capital Territory of Delhi, India.

\section{Competing Interests}

The authors declared that they have no competing interests.

\section{References}

[1] US Department of States (2012) 2012 International Narcotics Control Strategy Report. https://www.state.gov/j/inl/rls/nrcrpt/2012/vol1/184100.htm

[2] United Nations Convention against Illicit Traffic in Narcotic Drugs and Psychotropic Substances (1988). https://www.unodc.org/pdf/convention_1988_en.pdf

[3] Narcotics Control Bureau, India (2015) Annual Report 2015. http://narcoticsindia.nic.in/upload/download/document_id43e4e6a6f341e00671e12 3714de019a8.pdf

[4] The Narcotic Drugs and Psychotropic Substances Act, 1985 (61 of 1985) (1985). http://narcoticsindia.nic.in/upload/download/document_id08b2dbdc9ca941d23789 3bd425af8bfa.pdf 
[5] Beyerinck, M.W. (1889) Ein einfacher Diffusionsversuch. Zeitschrift für Physikalische Chemie, 3, 110-112.

[6] Grob, R.L. and Barry, E.F., Eds. (2004). Modern Practice of Gas Chromatography. John Wiley \& Sons, New York. https://doi.org/10.1002/0471651141

[7] Srivastava, M., Ed. (2010) High-Performance Thin-Layer Chromatography (HPTLC). Springer Science \& Business Media, New York.

[8] Pavia, D.L., Lampman, G.M., Kriz, G.S. and Vyvyan, J.A. (2008) Introduction to Spectroscopy. Cengage Learning, Boston.

[9] Logan, B.K., Stafford, D.T., Tebbett, I.R. and Moore, C.M. (1990) Rapid Screening for 100 Basic Drugs and Metabolites in Urine Using Cation Exchange Solid-Phase Extraction and High-Performance Liquid Chromatography with Diode Array Detection. Journal of Analytical Toxicology, 14, 154-159. https://doi.org/10.1093/jat/14.3.154

[10] Karasek, F.W. and Clement, R.E. (2012) Basic Gas Chromatography-Mass Spectrometry: Principles and Techniques. Elsevier, Amsterdam.

[11] Kalsi, P.S. (2007) Spectroscopy of Organic Compounds. New Age International, New Delhi.

[12] Alinejad, S., et al. (2016) Nephrotoxicity of Methadone: A Systematic Review. SpringerPlus, 5, 2087. https://doi.org/10.1186/s40064-016-3757-1

[13] https://www.asam.org/docs/default-source/advocacy/opioid-addiction-disease-facts -figures.pdf

[14] Verma. K.L., et al. (2016) Forensic Analysis of Narcotic Drugs-Challenges and Opportunities. Current Science, 111, 23-24.

[15] Jeremias, A. and Brown, D. (2010) Cardiac Intensive Care. 2nd Edition, Elsevier, Philadelphia.

[16] Leach, J.P. (2014) Antiepileptic Drug Therapy. In: Aminoff, M.J. and Daroff, R.B., Eds., Encyclopedia of the Neurological Sciences, 2nd Edition, Elsevier, Amsterdam, 224-229.

[17] Holstege, C.P. and Holstege, E. (2014) Caffeine. In: Wexler, P., Ed., Encyclopedia of Toxicology, 3rd Edition, Elsevier, Amsterdam, 617-620.

[18] Cortazzo, M.H., Copenhaver, D. and Fishman, S.M. (2014) Major Opioids and Chronic Opioid Therapy In: Benzon, H., Rathmell, J., Wu, C.L., Turk, D., Argoff, C. and Hurley, R., Eds., Practical Management of Pain, 5th Edition, Elsevier, Amsterdam, 495-507.

[19] Smith, H.S. (2014) Analgesics, Other. In: Aminoff, M.J. and Daroff, R.B., Eds., Encyclopedia of the Neurological Sciences, 2nd Edition, Elsevier, Amsterdam, 168-171.

[20] Enna, S.J. and Bylund, D.B. (2007) Alprazolam. In: Chaytor, A., Ed., xPharm: The Comprehensive Pharmacology Reference, Elsevier, Amsterdam, 1-4. http://www.sciencedirect.com/topics/neuroscience/alprazolam

[21] Kodaira, H. and Spector, S. (1988) Transformation of Thebaine to Oripavine, Codeine, and Morphine by Rat Liver, Kidney, and Brain Microsomes. Proceedings of the National Academy of Sciences, 85, 1267-1271. https://doi.org/10.1073/pnas.85.4.1267

[22] Heroin. Drugs Profile. https://www.drugabuse.gov/publications/drugfacts/heroin

[23] Douglas Bruce, R., Altice, F.L. and Friedland, G. (2012) HIV Disease among Substance Users: Treatment Issues. In: Sande's HIVIAIDS Medicine, 2nd Edition, El- 
sevier, Amsterdam, 537-549.

[24] DeMott, M., Young, M., Williams, S.R. and Clark, R.F. (2010) Management of commonly Encountered Drugs Poisoning. In: Jeremias, A. and Brown, D.L., Eds., Cardiac Intensive Care, 2nd Edition, Elsevier, Amsterdam, 401-402.

[25] Morphine Sulfate. The American Society of Health-System Pharmacists. https://www.drugs.com/monograph/morphine-sulfate.html

[26] Ebert, T.J. (2013) Autonomic Nervous System Pharmacology. In: Hemmings, H. and Egan, T., Eds., Pharmacology and Physiology for Anesthesia, Saunders, Philadelphia, 218-234.

[27] Verma, K.L., Choudhary, P., Sharma, M., Sarin, R.K. and Kumar, A. (2014) Identification of Heroin and Its Metabolite in Visceral Tissues of a Habitual Drug User-A Case Report. Journal of the Indian Society of Toxicology, 10, 18-22.

[28] Kasote, D., Ahmad, A., Chen, W., Combrinck, S. and Viljoen, A. (2015) HPTLC-MS as an Efficient Hyphenated Technique for the Rapid Identification of Antimicrobial Compounds from Propolis. Phytochemistry Letters, 11, 326-331.

https://doi.org/10.1016/j.phytol.2014.08.017

[29] De Castro, A., Concheiro, M., Shakleya, D.M. and Huestis, M.A. (2009) Simultaneous Quantification of Methadone, Cocaine, Opiates, and Metabolites in Human Placenta by Liquid Chromatography-Mass Spectrometry. Journal of Analytical Toxicology, 33, 243-252. https://doi.org/10.1093/jat/33.5.243

[30] Wankhede, S.B. and Chitlange, S.S. (2013) A Novel HPTLC Method for Simultaneous Determination of Alprazolam and Methyl Paraben in Tablet Dosage Form. Der Pharmacia Lettre, 5, 89-93.

[31] Qiao, H., Ye, J.S. and Hao, C. (2017) A Quantitative Determination of Methadone and Its Metabolite (EDDP) in Dry Blood Spot by LC-MS/MS. 62nd ASMS Conference on Mass Spectrometry and Allied Topics, Baltimore, 15-19 June 2014, IONICS Mass Spectrometry Group Inc., Bolton. http://gala.gre.ac.uk/12156/1/12156_asms-62nd_program_\%28June2014\%29.pdf

[32] Verma, K.L., Verma, R., Panicker, V., Sharma, M., Sarin, R.K. and Jaiswal, A.K. (2015) Determination of Ketamine by LC-MS/MS: A Method Validation for Forensic Investigation. UJP, 4, 72-79.

[33] Moffat, A.C., Osselton, M.D. and Widdop, B. (2003) Clarke's Analysis of Drugs and Poisons. 3rd Edition, Pharmaceutical Press, London. 